Pacific Journal of Mathematics

AN INTERPOLATION THEOREM IN THE PREDICATE 


\section{AN INTERPOLATION THEOREM IN THE PREDICATE CALCULUS}

\section{ROGER C. LYNDON}

1. Introduction. In studying the formal structure of sentences whose validity is preserved under passage from an algebraic system to a homomorphic image of the system, we have had occasion to use a lemma from formal logic. A proof of this lemma, our Interpolation Theorem, can be given within the theory of deductive inference, as formalized by Gentzen. Gentzen's theory is rather complicated and perhaps not generally well known. Moreover, the use of any formalized system of deductive logic seems to an extent alien to the primarily algebraic nature of our intended application. Therefore we give here a proof of the Interpolation Theorem that lies entirely within the theory of models : our arguments are as far as possible in the spirit of abstract algebra, and, in particular, borrow nothing from formal logic beyond an understanding of the intended meaning, herein precisely defined, of the conventional symbolism.

The Interpolation Theorem deals with sentences of the Predicate Calculus. Roughly, these are sentences that can be build up using the usual logical connectives, symbols denoting operations (or functions), symbols denoting relations (or predicates), and variables whose range is individual elements of the systems under consideration, but no variables ranging over operations, relations, or sets. The theorem takes the same form whether or not we admit a predicate denoting identity, with suitable axioms, to the predicate calculus. For technical reasons we admit as sentential connectives only the signs for negation, conjunction and disjunction (regarding "if ... then" as a defined concept), together with signs 0 and 1 for truth and falsehood. For each occurrence of a relation symbol in a sentence $S$, there is a unique maximal chain of well formed formulas, all containing the given occurrence and each occurring as a proper part of the next. The given occurrence of the relation symbol will be called positive if the number of formulas in this chain that begin with the negation sign is even, and negative if this number is odd. If $S$ is in prenex disjunctive form, this criterion takes the simpler form that an occurrence is negative if and only if it is preceded by the negation sign.

INTERPOLATION THEOREM, Let $S$ and $T$ be sentences such that $S$ implies $T$. Then there exists a sentence $M$ such that $S$ implies $M$ and $M$

Received October 22, 1958. Work supported in part under grants from the National Science Foundation,

1. See [5] and [9], Chapter XV. 
implies $T$, and that a relation symbol has positive occurrences in $M$ only if it has positive occurrences in both $S$ and $T$, and has negative occurrences in $M$ only if it has negative occurrences in both $S$ and $T$.

This theorem is a generalization of a result of $\mathrm{W}$. Craig [3, 4]; Craig's lemma is obtained from it by suppressing the distinction between positive and negative sentences. As indicated, our first proof of the Interpolation Theorem used the Gentzen calculus; it did not differ essentially from Craig's proof, at that time unpublished, of his lemma.

The leading idea of the present proof is to interpret $S$ implies $T$ to mean that $T$ holds in every model for which $S$ holds; we express this relation by writing $S \Rightarrow T$. By Gödel's Completeness Theorem [6], this semantic interpretation is equivalent to the interpretation $S \vdash T$, that $T$ is a formal consequence of $S$ in a deductive axiomatization of the predicate calculus. The crucial point in our argument is the Main Theorem, which serves as a substitute, under this interpretation, for results in the theory of proof due to Herbrand [8] and to Gentzen [5].

A theorem of the theory of proof may be taken, in general, as saying that if there exists any derivation of one set $\Delta$ of formulas from a set $\Gamma$, then there exists a derivation with certain special properties. A semantic counterpart of such a theorem will take the form of an 'interpolation theorem' : if $I^{\prime} \Rightarrow \Delta$, then there exists a chain $\Gamma=\Gamma^{1}$, $\Gamma^{2}, \cdots, \Gamma^{n}=\Delta$ of sets of formulas, with certain special properties, such that $\Gamma^{1} \Rightarrow \Gamma^{2}, \cdots, \Gamma^{n-1} \Rightarrow \Gamma^{n}$. Theorems of this sort will ordinarily require the occurrence in the $\Gamma^{k}$ of additional symbols (for the 'Skolem functions') that do not appear in $\Gamma$ or $\Delta$, although this is not true of the Interpolation Theorem. Our arguments abjure any formal use of the concept of deductive derivablilty, hence of the Completeness Theorem. In various special cases, where $\Gamma \vdash \Delta$ would be immediate, that $\Gamma \Rightarrow \Delta$ follows directly from our definitions. The more difficult half of the Completeness Theorem, that if $\Gamma \Rightarrow \Delta$ then $\Gamma \vdash \Delta$, is implicit in the Main Theorem, which guarantees the existence of a chain $\Gamma=\Gamma^{\perp}, \cdots, \Gamma^{n}=\Delta$ such that at each step the relation $\Gamma^{k} \vdash \Gamma^{k+1}$ is immediately evident.

I have profited much from discussions related to the present topic with A. Tarski and L. Henkin ${ }^{2}$; in particular, Tarski has emphasized the desirability of establishing the Interpolation Theorem by methods independent of the theory of proof. The idea of providing semantic proofs of results from the theory of proof is not new : a proof by E. Beth [1, 2], in a quite different formalism, of Craig's Lemma would certainly serve as well to prove the Interpolation Theorem; and A. Robinson has likewise provided semantic proofs of closely related results [10]. Unpublished results similar to those presented here have recently been

2 In particular, while the author was visiting at the University of California, Berkeley. 
obtained by A. Grzegorczyk, A. Mostowski and C. Ryll-Nardzewski, and by R. Vaught.

2. Basic concepts. ${ }^{3}$ A language $L$ is determined by an ordered quadruple, $V, W, R, \rho$, where $V, W, R$ are disjoint sets, $V$ infinite, and $\rho$ is a function from $W \cup R$ to the natural numbers. The elements of $V$ will be called variables, those of $W$ operation symbols, and those of $R$ relation symbols; for $w$ in $W, r$ in $R, \rho(w)$ is the rank of $w$ and $\rho(r)$ the rank of $r$. The logical symbols are $0,1, \sim, \wedge \vee, \forall, \exists$. The expressions of $L$ will be made up of these symbols together with parentheses and commas. A term is, recursively, any variable, and any expression $w\left(t_{1}, \cdots, t_{\rho(w)}\right)$ where $w$ is an operation symbol and $t_{1}, \cdots, t_{\rho(w)}$ are terms. An atomic formula is any expression $r\left(t_{1}, \cdots, t_{\rho(r)}\right)$ where $r$ is a relation symbol and $t_{1}, \cdots, t_{\rho(r)}$ are terms. A formula is, recursively, any atomic formula, and any expression $0,1, \sim F,(F \wedge G),(F \vee G), \forall x F, \exists x F$ where $F$ and $G$ are formulas and $x$ is a variable. Formally, we define $L$ to be the set of its symbols, terms and formulas.

We introduce the abbreviations $F \supset G$ for $(\sim F \vee G), \bigwedge_{1}^{n} F_{i}$ for $F_{1} \wedge \cdots \wedge F_{n}$ with the convention $\Lambda_{1}^{0} F_{i}=1$, and $\bigvee_{1}^{n} F_{i}$ for $F_{1} \vee \cdots \vee F_{n}$ with $V_{1}^{0} F_{i}=0$, and write $\forall x_{1} \cdots x_{n}$ for $\forall x_{1} \cdots \forall x_{n}$. A matrix is a formula that does not contain $\forall$ or $\exists$. A normal matrix is a matrix of the form $\bigvee_{i=1}^{m} \bigwedge_{j=1}^{n_{m}} F_{i j}$ where each $F_{i j}$ is either $A_{i j}$ or $\sim A_{i j}$, for $A_{i j}$ an atomic formula. A prenex formula is one of the form $Q_{1} x_{1} \cdots Q_{q} x_{q} M$ where each $Q_{i}$ is $\forall$ or $\exists$, each $x_{i}$ is a variable, and $M$ is a matrix ; the formula is normal if the matrix $M$ is normal. An occurrence of a variable $x$ in a formula $F$ is free in the formula $F$ if it is not part of a subformula of the forms $\forall x G$ or $\exists x G$. A sentence is a formula without free occurrences of variables.

It is easily shown by induction that if $G$ is any part of a formula $F$, then there is a smallest part of $F$ that is a formula and contains $G$. It follows that there is a unique maximal chains of formulas $H_{1}, \cdots, H_{n}=F$, each a proper part of the next, and all containing $G$. The part $G$ is positive in $F$ if the number of $H_{i+1}=\sim H_{i}$ is even, and negative if it is odd. In what follows, $G$ will always be an occurrence of a relation symbol in $F$.

An interpretation of a language $L$ is determined by a set $A$ and a function $\mu$, defined on $V \cup W \cup R$, such that $\mu x \in A$ for $x \in V, \mu w \in A^{A^{\rho(w)}}$ for $w \in W$, and $\mu r \in 2^{4^{(p(r)}}$ for $r \in R$. We regard 2 as the two element Boolean algebra with elements 0,1 and operations $\sim, \wedge, \vee$, so that $\mu r$ is a function with values $\left(\mu r^{\circ}\right)\left(a_{1}, \cdots, a_{p(r)}\right)$ equal to 0 or 1 ; but in practice we indulge in the harmless ambiguity of treating $\mu w$ as a subset of $A^{\rho(w)+1}$ 
and $\mu r$ of $A^{\rho(r)}$, and accordingly using such notation as $\mu w \subseteq \mu w^{\prime}, \mu r \subseteq \mu r^{\prime}$. Putting aside the trivial case that $L$ contains no relation symbols of positive rank, $\mu$ unambiguously determines its domain $A$.

The function $\mu$ determines a unique extension mapping all terms of $L$ into $A$, by the recursive definition

$$
\mu\left[w\left(t, \cdots, t_{\rho(w)}\right)\right]=(\mu w)\left(\mu t, \cdots, \mu t_{\rho(w)}\right) .
$$

A further extension mapping all formulas of $L$ into 2 is determined by the conditions.

$$
\begin{gathered}
\mu 0=0, \mu 1=1, \quad \mu(\sim F)=\sim \mu F, \quad \mu(F \wedge G)=\mu F \wedge \mu G, \\
\mu(F \vee G)=\mu F \vee \mu G,
\end{gathered}
$$

and

$$
\left.\begin{array}{lll}
\mu(\forall x F)=1 & \text { if and only if } \lambda F=1 \text { for all } \lambda \\
\mu(\exists x F)=1 & \text { if and only if } \lambda F=1 \text { for some } \lambda
\end{array}\right\} \text { such that }
$$

$\lambda z=\mu z$ for all $z$ in $V \cup W \cup R-\{x\}$. Formally, we define an interpretation to be a function $\mu$ thus extended; in practice we shall say that

$\mu$ and $\lambda$ agree except on $x$ when we mean that $\mu$ and $\lambda$ agree for all $z$ in $V \cup W \cup R-\{x\}$.

A model of $L$ is the restriction $\mathfrak{A}$ of an interpretation $\mu$ to the operation and relation symbols of $L$. The model $\mathfrak{A}$ may be regarded as a 'relational system" ${ }^{4}$ consisting of a set $A$, its domain, together with a set of operations $\mathfrak{A} w$ indexed by the operation symbols $w$ of $L$, and a set of relations $\mathfrak{A} r$ indexed by the relation symbols $r$ of $L$. If $\mathfrak{A}$ is the restriction of $\mu$, we call $\mu$ an interpretation in the model $\mathfrak{A}$. If $\mu F=1$, we say that $F$ holds for the interpretation $\mu$. Evidently $\mu F$ depends only on the domain $A$ of $\mu$, the values of $\mu$ on the operation and relation symbols that occur in $F$, and the values of $\mu$ on the variables that occur free in $F$. In particular, if $S$ is a sentence, $\mu S$ depends only on the model $\mathfrak{A}$ to which $\mu$ belongs, and if $\mu S=1$ we say that $S$ holds in the model $\mathfrak{A}$.

If $\Gamma$ and $\Delta$ are sets of formulas of $L$, we say that $\Gamma^{\prime}$ implies $\Delta$ in $L$ if $\mu \Delta=\{1\}$ for all interpretations of $L$ such that $\mu \Gamma=\{1\}$. This interpretation is evidently independent of $L$, provided only that $\Gamma$ and $\Delta$ belong to $L$; we say simply that $\Gamma$ implies $\Delta$, and write $\Gamma \Rightarrow \Delta$. We write $\mu \Gamma=1$ for $\mu \Gamma^{\prime}=\{1\}$, and employ such notation as $\Gamma_{1}, \Gamma_{2} \Rightarrow F$ with the obvious meaning. If $\Gamma \Rightarrow \Delta$ and $\Delta \Rightarrow \Gamma$, then $\Gamma$ and $\Delta$ are equivalent and we write $\Gamma \Leftarrow \Rightarrow \Delta$. That $1 \Rightarrow F$ expresses that $F$ is a theorem. A set $\Gamma$ is called consistent if there exists an interpretation $\mu$ such that $\mu \Gamma=1$; thus $\Gamma \Rightarrow 0$ expresses that the set $\Gamma$ is inconsistent.

${ }^{4}$ See $[\mathbf{1 1}],[12]$. 
3. Preliminary propositions. The set $\Phi=\Phi(L)$ of all formulas of $L$ constitutes, in an obvious sense, an algebraic system with operations $0,1, \sim, \wedge, \vee$; in fact it is a 'word algebra', a free algebra without axioms. The relation $F \Leftrightarrow G$ is a congruence on $\Phi$, and the quotient system $\bar{\Phi}$ is a Boolean algebra, the Lindenbaum algebra of $L$. If $\kappa$ is the canonical map of $\Phi$ onto $\bar{\phi}$, then every interpretation $\mu$ of $L$, when restricted to $\Phi$, can be factored uniquely in the form $\mu=\bar{\mu}_{\kappa}$ where $\bar{\mu}$ is a homomorphism of $\bar{\phi}$ onto 2 .

The set $\Phi_{0}$ of all matrices of $L$ constitutes a subalgebra of $\Phi$, and its image $\overline{\Phi_{0}}=\kappa \Phi_{0}$ is a subalgebra of $\bar{\Phi}$. Every homomorphism $\theta$ of $\bar{\Phi}_{0}$ onto 2 can be extended to a homomorphism $\theta^{\prime}$ of $\bar{\Phi}$ onto 2 such that $\theta^{\prime} \kappa$ is an interpretation. To prove this we construct the special interpretation $\mu$ induced by $\theta$. For the domain $A$ of $\mu$ we take the set of all terms of $L$. For a variable $x$, define $\mu x=x$. For an operation symbol $w$ and terms $t_{1}, \cdots, t_{\rho(w)}$, we define $\mu w$ by assigning to $(\mu w)\left(t_{1}, \cdots, t_{\rho(w)}\right)$ as value the term $w\left(t_{1}, \cdots, t_{\rho(w)}\right)$. For a relation symbol $r$ and terms $t_{1}, \cdots, t_{\rho(r)}$ we define $\mu r$ by assigning to $(\mu r)\left(t_{1}, \cdots, t_{\rho(r)}\right)$ the value $\theta \kappa\left[r\left(t_{1}, \cdots, t_{\rho(r)}\right)\right]$ in 2. By virtue of the last definition, $\mu F=\theta \kappa F$ for all atomic formulas $F$. Since the images $\kappa F$ of the atomic formulas $F$ generate $\bar{\Phi}_{0}$, and $\bar{\mu} \kappa F=\theta \kappa F$ for atomic $F$, it follows that $\bar{\mu}=\theta$ on $\bar{\Phi}_{0}$ and $\bar{\mu}$ is an extension of $\theta$.

Proposition 1. If $\Gamma$ is a set of matrices, and $J$ the dual ideal in the Boolean algebra $\bar{\Phi}_{0}$ generated by $\kappa \Gamma$, then $\Gamma \Rightarrow 0$ if and only if $0 \in J$.

Proof. Assume $0 \in J$. Then $0=\kappa F_{1} \wedge \cdots \wedge \kappa F_{n}$ for some $F_{1}, \cdots, F_{n}$ in $\Gamma$. If $\mu$ is an interpretation such that $\mu I^{\prime}=1$, then each $\bar{\mu}_{\kappa} F_{i}=\mu F_{i}=1$, whence $1=\bar{\mu} \kappa \wedge F_{i}=\bar{\mu} \wedge \kappa F_{i}=\bar{\mu} 0=0$, a contradiction. Assume $0 \notin J$. Then $J \neq \Phi_{0}$ and $J \subseteq K$ for some maximal dual ideal $K$ in $\overline{\Phi_{0}}$ If $\theta$ is the canonical map of $\Phi_{0}$ onto 2 with kernel the maximal ideal $\overline{\Phi_{0}}-K$ complementary to the dual ideal $K$, then $\theta \kappa \Gamma \subseteq \theta J \subseteq \theta \kappa=1$. If $\mu$ is the special interpretation of $L$ induced by the homomorphism $\theta$, then $\mu \Gamma=\bar{\mu} k \Gamma=\theta \kappa \Gamma=1$, whence $\Gamma$ is consistent.

COROLlary 1.1. If $\Gamma$ is a set of matrices, then $\Gamma \Rightarrow 0$ if and only if $\Gamma_{0} \Rightarrow 0$ for some finite subset $\Gamma_{0}$ of $\Gamma$.

Every map $\sigma$ of the atomic formulas of $L$, as free generators of $\Phi_{0}$, into $\Phi_{0}$, extends to an endomorphism of $\Phi_{0}$, which in turn induces an endomorphism $\bar{\rho}$ of $\bar{\Phi}_{0}$. It follows that if $\Gamma \Rightarrow 0$ then $\sigma \Gamma \Rightarrow 0$. Every map $\sigma$ of the variables of $L$ into terms of $L$ extends in an obvious way to a map of the terms of $L$ into terms of $L$, hence of formulas of $L$ into formulas of $L$; a transformation induced in this fashion will be 
called a substitution.

Proposition 2. Let $\Gamma$ be set of sentences $S$ of the form $\forall x_{1} \cdots x_{n} M$ where the $M$ are matrices, and $\Gamma^{\prime}$ the set of all formulas $\sigma M$ where $\sigma$ is a substitution and $M$ is the matrix of some sentence $S$ in $\Gamma$. Then $I \Rightarrow 0$ if and only if $\Gamma^{\prime} \Rightarrow 0$.

Proof. Suppose that $\Gamma^{\prime}$ is consistent. Then $\lambda \Gamma^{\prime}=\bar{\lambda} k \Gamma^{\prime}=1$ for some interpretation $\lambda$. Let $\mu$ be the special interpretation induced by the homomorphism $\lambda$ of $\Phi_{0}$ onto 2 . Let $F=\forall x_{1} \cdots x_{n} M$ be in $I^{\prime}$, and $\nu$ be an interpretation that agrees with $\mu$ except on $x_{1}, \cdots, x_{n}$. Since the values $\nu x$ for variables $x$ are terms, we may define a substitution by setting $\sigma x=\llcorner x$. Since $\mu x=x$ for all variables $x, \nu M=\mu \sigma M=\bar{\lambda} \sigma M=1$. This establishes that $\mu F=1$. Suppose $\Gamma^{\prime}$ is inconsistent. Then for all interpretations $\mu$ there is some $F=\forall x_{1} \cdots x_{n} M$ in $I^{\top}$ and some substitution $\sigma$ such that $\mu \sigma M=0$. Then setting $\lambda x_{i}=\mu \sigma x_{i}, i=1, \cdots, n$ defines an interpretation $\lambda$ that agrees with $\mu$ except on $x_{1}, \cdots, x_{n}$, and such that $\lambda M=0$. It follows that $\mu F=0$.

Corollary 2.1. If $I$ is a set of universal sentences, of the form $F=\forall x_{1} \cdots x_{n} M$, where $M$ is a matrix, then $\Gamma \Rightarrow 0$ if and only if $\Gamma_{0} \Rightarrow 0$ for some finite subset $I_{0}$ of $I^{\prime}$.

A prenex sentence $S$ of the language $L$ may be written in the form

$$
S=\forall x_{11} \cdots x_{1 m_{1}} \exists y_{1} \cdots \forall x_{n 1} \ldots x_{n m_{n}} \exists y_{n} M
$$

where $n, m_{1}, \cdots, m_{n}$ are natural numbers, the $x_{p q}$ and $y_{r}$ are variables, and $M$ is a matrix. The Skolem matrix of $S$ is the result $\sigma M$ of substituting $\sigma y_{r}=s_{r}\left(x_{11}, \cdots, x_{r m_{r}}\right)$ and $\sigma z=z$ for all other variables $z$; here the $s_{1}, \cdots, s_{n}$ are new and distinct operation symbols which we may suppose uniquely associated with the pair consisting of $S$ and $L$. The Skolem form of $S$ is the sentence $\forall x_{11} \cdots x_{n m_{n}} \sigma M$. The Skolem form belongs to the language $L^{\prime}$ obtained by adjoining the symbols $s_{1}, \cdots, s_{n}$ to $L$.

LEMMA 3. Let $S$ be a sentence of the form

$$
S=\forall x_{11} \cdots x_{m_{1}} \exists y_{1} \cdots x_{n 1} \forall \cdots x_{n m_{n}} \exists y F,
$$

where the $x_{p q}$ and $y_{r}$ are distinct variables and $F$ is a formula in which all occurrences of these variables are free. Let $F^{\prime \prime}$ result from $F$ by substituting for each $y_{r}$ a term $\sigma y_{r}$ that contains no variables othar than $x_{11}, \cdots, x_{r m_{r}}$. Let $S^{\prime}$ be the sentence

$$
S^{\prime}=\forall x_{11} \cdots x_{1 m_{1}} x_{i 1} \cdots x_{n m_{n}} F^{\prime} .
$$


Then $S^{\prime} \Rightarrow S$.

Proof $^{5}$. We proceed by induction. For $n=0$ the assertion is trivial. For $n=1$ it suffices to observe that if $\mu$ is an interpretation such that $\mu F^{\prime}=1$, then defining an interpretation $\lambda$ to agree with $\mu$ except on $y_{1}$, and setting $\lambda y_{1}=\mu \sigma y_{1}$, gives $\lambda F=\mu F^{\prime}$, hence $\lambda F=1$. For $n>1$, form $F^{\prime \prime}$ from $F$ by substituting $\sigma y_{r}$ for $y_{r}$, all $y_{r}$ except $y_{n}$, and let $S^{\prime \prime}=$ $\forall x_{11} \cdots x_{n m_{n}} \exists y F^{\prime \prime}$. Then the case $n=1$ applies to give $S^{\prime} \Rightarrow S^{\prime \prime}$, and the case $n-1$ to give $S^{\prime \prime} \Rightarrow S$.

Proposition 4. Let $\Gamma$ be a set of prenex sentences of a language $L$, and $\Gamma^{\prime}$, in an extended language $L^{\prime}$, the set of all Skolem forms of the sentences in $\Gamma$. Then $\Gamma$ holds in a model $\mathfrak{A}$ of $L$ if and only if $\Gamma^{\prime}$ holds in some extension $\mathfrak{Y}^{\prime}$ of $\mathfrak{A}$ to a model of $L^{\prime}$.

Proof. By an induction it evidently suffices to establish the conclusion under the assumption that $\Gamma^{\prime}$ results from $\Gamma$ by replacing a single sentence $S$ by its Skolem form $S^{\prime}$. If $\Gamma^{\prime}$ holds in an extension $\mathfrak{U}^{\prime}$ of $\mathfrak{Y}$ to $L^{\prime}$, it follows by Lemma 3 that $\Gamma$ holds in $\mathfrak{Y}^{\prime}$, and, since $\Gamma$ belongs to $L$, that $\Gamma$ holds in $\mathfrak{Y}$. For the rest, by a second induction it suffices to establish the conclusion for $S=\forall x_{1}, \cdots, x_{m} \exists y F, S^{\prime}=\forall x_{1} \cdots x_{m} \sigma F$, $F$ a formula, $\sigma x_{i}=x_{i}, i=1, \cdots, m$, and $\sigma y=s\left(x_{1}, \cdots, x_{m}\right)$, where $s$ does not belong to $L$ and $L^{\prime}$ is obtained by adjoining $s$ to $L$.

Assume now that $\Gamma$ holds in $\mathfrak{P}$. For any $a_{1}, \cdots, a_{m}$ in the domain $A$ of $\mathfrak{A}$, there exists an interpretation $\mu$ in $\mathfrak{A}$ such that $\mu x_{i}=a_{i}, i=1, \cdots, m$. Since $\mu S=1$, it follows that $\mu(\exists y F)=1$, and there exists an interpretation $\lambda$ that agrees with $\mu$ except on $y$ such that $\lambda F=1$. By the axiom of choice we may define a function $f$ from $A^{m}$ into $A$ by choosing for all $\alpha, \cdots, a_{m}$ interpretations $\mu$ and $\lambda$ as above and setting $f\left(a_{1}, \cdots, a_{m}\right)=\lambda y$. Extend $\mathfrak{V}$ to $\mathfrak{H}^{\prime}$ by defining $\mathfrak{U}^{\prime} \mathrm{s}=f$. If $\mu^{\prime}$ is an interpretation in $\mathfrak{X}^{\prime}$, then $\mu^{\prime}$ agrees with some $\mu, \lambda$ as above on the variables $x_{1}, \cdots, x_{m}$. Moreover, $\mu^{\prime} \sigma y=f\left(\mu^{\prime} x_{1}, \cdots, \mu^{\prime} x_{m}\right)=f\left(a_{1}, \cdots, a_{m}\right)=\lambda y$, whence $\mu^{\prime} \sigma F=$ $\lambda F=1$. It follows that $\mu^{\prime} S^{\prime} 1$ for all interpretations $\mu^{\prime}$ in $\mathfrak{Y}^{\prime}$, whence $\Gamma^{\prime}$ holds in $\mathfrak{X}^{\prime}$.

COROLlary 4.1. If $\Gamma$ is any set of prenex sentences, then $\Gamma \Rightarrow 0$ if and only if $\Gamma_{0} \Rightarrow 0$ for some finite subset $\Gamma_{0}$ of $\Gamma$.

Every sentence is equivalent to a prenex sentence, and, indeed, a normal sentence. This follows by induction from various immediate consequences of the definitions, of which $\sim(F \wedge G) \Leftrightarrow(\sim F \vee \sim G)$ and $\forall x(F \wedge G) \Leftrightarrow(\forall x F \wedge \forall x G)$ are typical. In fact, it is easily seen that

${ }^{5}$ C. C. Chang pointed out to me a gap in an earlier version of this proof. 
every sentence $S$ is equivalent to a normal sentence $S^{\prime}$ such that a relation symbol occurs positively (negatively) in $S^{\prime}$ only if it occurs positively (negatively) in $S$.

In view of this, Corollary 4.1 yields the Compactness Theorem.

Proposition 5. If $\Gamma$ is any set of sentences, then $\Gamma \Rightarrow 0$ if and only if $\Gamma_{0} \Rightarrow 0$ for some finite subset $\Gamma_{0}$ of $\Gamma$.

4. The main theorem. Let $S$ be a prenex sentence, of the form

$$
S=\forall x_{11} \ldots \forall \forall_{1 m_{1}} \exists y_{1} \cdots \forall x_{n 1} \ldots x_{n m_{n}} \exists y_{n} M .
$$

A second sentence $S_{0}$ will be said to arise from $S$ by duplication if

(i) $\pi_{1}, \cdots, \pi$ are substitutions such that all $\pi_{i} x_{p q}=x_{p q}^{i}, \pi_{i} y_{r}=y_{r}^{i}$, where the $x_{p q}^{i}$ and $y_{r}^{i}$ are distinct variables; and

(ii) $S_{0}$ results from $\pi_{1} M \wedge \cdots \wedge \pi_{a} M$ by prefixing quantifiers $\forall x_{p q}^{i}$ and $\exists y_{r}^{i}$ in some order such that, for $p \leqq r, \forall x_{p q}^{i}$ precedes $\exists y_{r}^{i}$.

Proposition 6. If $S_{0}$ arises from $S$ by duplication, then $S \Rightarrow S_{0}$.

Proof. Let $S$ have Skolem matrix $\sigma M$, in the language $L^{\prime}$, where $\sigma x_{p q}=x_{p q}$ and $\sigma y_{r}=s_{r}\left(x_{11}, \cdots x_{r m_{r}}\right)$. By Proposition 4, if $S$ holds in any model $\mathfrak{U}$, then its Skolem form $S^{\prime}$ holds in some extension $\mathfrak{H}^{\prime}$ of $\mathfrak{A}$ to $L^{\prime}$. If $\mu$ is an interpretation of $L^{\prime}$ in $\mathfrak{H}^{\prime}$, then every substitution instance of $\sigma M$ holds in $\mu$; in particular, all $\pi_{i} \sigma M$ hold in $\mu$, whence $\Lambda \pi_{i} \sigma M$ holds in $\mu$. But $\Lambda \pi_{i} \sigma M$ results from $\Lambda \pi_{i} M$ by substituting $s_{r}\left(x_{11}^{i}, \cdots, x_{r m_{r}}^{i}\right)$ for each $y_{r}^{i}$, whence, by Lemma $3, S_{0}$ holds in $2{ }^{\prime}$, and therefore in $\mathfrak{U}$.

For $S$ as before, a second sentence $S_{0}$ will be said to arise from $S$ by specialization if

(iii) $\theta$ is a substitution such that $\theta y_{r}=y_{r}$, while each $\theta x_{p q}$ is a term in certain new variables $u_{1} \cdots, u_{a}$ together with the $y_{r}$ for $r<p$; and

(iv) $S_{0}$ results from $\theta M$ by prefixing quantifiers $\forall u_{h}$ and $\exists y_{r}$ in some order such that $\forall u_{h}$ precedes $\exists y_{r}$ if $u_{h}$ occurs in any $\theta x_{p q}$ for $p \leqq r$, and $\exists y_{s}$ precedes $\exists y_{r}$ if $y_{s}$ occurs in any $\theta x_{p q}$ for $p \leqq r$.

Proposition 7. If $S_{0}$ arises from $S$ by specialization, then $S \Rightarrow S_{0}$.

Proof. Let $S$ have Skolem matrix $\sigma M$ in $L^{\prime}$ as before. Define a substitution $\rho$ by setting $\rho z=z$ for all variables $z$ other than the $y_{r}$, and, by recursion on the order of quantification of the $y_{r}$ in $S_{0}$, defining $\rho y_{r}=\rho \theta \sigma y_{r}=s_{r}\left(\rho \theta x_{11}, \cdots, \rho \theta x_{r m_{r}}\right)$. Since all $y_{s}$ that occur in $\theta \sigma y_{r}$ occur in some $\theta x_{p q}$ for $p \leqq r$, all such $y_{s}$ precede $y_{r}$ in $S_{0}$, and the recursion 
if legitimate. Since $\theta y_{r}=y_{r}, \rho \theta y_{r}=\rho y_{r}=\rho \theta \sigma y_{r}$ by the above definition, while for all other variables $z, \sigma z=z$ and again $\rho \theta z=\rho \theta \sigma z$. Suppose now that $S$ holds in a model $\mathfrak{A}$ of $L$, and hence, by Proposition 4 , that the Skolem form $S^{\prime}$ of $S$ holds in an extension $\mathfrak{A}^{\prime}$ of $\mathfrak{A}$ to $L^{\prime}$. Then, for every interpretation $\mu$ in $\mathfrak{U}^{\prime}$, all instances of $\sigma M$ hold, and, in particular, $\rho \theta_{\sigma} M$ holds. Since $\rho \theta \sigma=\rho \theta, \rho \theta \sigma M=\rho \theta M$. Now $\rho \theta M$ results from $\theta M$ by the substitution $\rho$, and $\rho u_{h}=u_{h}$, while $\rho y_{r}$ contains only those $u_{h}$ that occur in the $\rho \theta x_{p q}$ for $p \leqq r$; by induction, using (iii), these are among the $u_{l}$ that occur in $\theta x_{p q}$ for $p \leqq r$, and hence among the $u_{h}$ that precede $y_{r}$ in $S_{0}$. Therefore Lemma 3 applies to establish that $S_{0}$ holds in $\mathfrak{U}^{\prime}$ and thus in $\mathfrak{A}$.

Let $S^{1}, S^{2}$ be prenex sentences of the form, for $\delta=1,2$,

$$
S^{\delta}=\forall x_{11}^{\delta} \cdots x_{1 m_{1}}^{\delta} \exists y_{1}^{\delta} \cdots \forall x_{n 1}^{\delta} \cdots x_{n m_{n}}^{\delta} \exists y_{n}^{\delta} M^{\delta}
$$

with Skolem matrices $\sigma M^{\delta}$ in a language $L^{\prime}$, where $\sigma x_{p q}^{\delta}=x_{p q}^{\delta}, \sigma y_{r}^{\delta}=$ $s_{r}^{\delta}\left(x_{11}^{s}, \cdots x_{r_{r}}^{\delta}\right)$. Then $S^{1}$ and $S^{2}$ will be called propositionally inconsistent if there exists a substitution $\eta$ in $L^{\prime}$ that is one-to-one on all atomic formulas of each $\sigma M^{\delta}$ such that $\eta \sigma M^{1}, \eta \sigma M^{2} \Rightarrow 0$.

Proposition 8. If $S^{1}, S^{2}$ are propositionally inconsistent, then $S^{1} S^{2} \Rightarrow 0$.

Proof. Suppose $S^{1}, S^{3}$ were consistent, hence both held in some model $\mathfrak{i}$ of $L$. Using Proposition 4, all instances of $\sigma M^{1}$ and $\sigma M^{2}$ would hold for all interpretations in a certain extension $\mathfrak{U}^{\prime}$ of $\mathfrak{U}$ to a model of $L^{\prime}$. Then $\eta \sigma M^{1}$ and $\eta \sigma M^{2}$ would hold for all such $\mu$, and $\mu 0=1$, a contradiction.

In propositions 6,7 and 8 we have attempted to isolate the chief ideas that underly the Main theorem; the proof of this theorem can now be accomplished by easier and more natural stages, although at the cost of a small amount of repetition.

MaIN Theorem. Let $S^{1}$ and $S^{2}$ be prenex sentences such that $S^{1}, S^{2} \Rightarrow 0$. Then there exist prenex sentences $T^{\perp}, T^{2}, U^{1}$ and $U^{2}$ such that (1) $T^{1}$ arises from $S^{1}$, and $T^{2}$ from $S^{2}$, by duplication; (2) $U^{1}$ arises from $T^{1}$, and $U^{2}$ from $T^{2}$, by specialization; and (3) $U^{1}$ and $U^{2}$ are propositionally inconsistent.

Proof. Let $S^{1}, S^{2}, M^{1}, M^{2}, \sigma$ and $L, L^{\prime}$ be as above. (There is clearly no loss of generality in taking common values of $n$ and the $m_{r}$, and a common substitution $\sigma$, for $S^{1}$ and $S^{2}$.) By Proposition $4, S^{1}, S^{2} \Rightarrow 0 \mathrm{im}$ plies that their Skolem forms are inconsistent. By Proposition 2, the set of all instances of $\sigma M^{1}$ and $\sigma M^{2}$ is consistent. By Corollary 1.1 some finite set of these instances is inconsistent. Therefore there exist substitutions $\eta_{1}, \cdots, \eta_{a}$ in the language $L^{\prime}$ such that 


$$
\eta_{1} \sigma M^{1}, \cdots, \eta_{a} \sigma M^{1}, \eta_{1} \sigma M^{2}, \cdots, \eta_{a} \sigma M^{2} \Rightarrow 0
$$

Define substitutions $\pi_{1}, \cdots, \pi_{a}$ such that all $\pi_{i} x_{p q}^{\delta}=x_{p q}^{\delta i}$ and $\pi_{i} y_{r}^{\delta}=y_{r}^{\delta i}$, where the $x_{r q}^{\delta i}$ and $y_{r}^{\delta i}$ are new and distinct variables. Define $\sigma^{\prime}$ such that $\sigma^{\prime} x_{p q}^{\delta i}=x_{p q}^{\delta i}$ and $\sigma^{\prime} y_{r}^{\delta i}=s_{r}^{\delta}\left(x_{11}^{\delta i}, \cdots, x_{r m_{r}}^{\delta i}\right)$; thus $\sigma^{\prime} \pi_{i} M^{\delta}=\pi_{\iota} \sigma M^{\delta}$ for all $\pi_{i}$. Define $\eta$ such that $\gamma_{i} x_{p q}^{\delta i}=\gamma_{i} x_{i q}^{\delta}$; then $\eta \sigma^{\prime} \pi_{i} M^{\delta}=\eta \pi_{i} \sigma M^{\delta}=\eta_{i} \sigma M^{\delta}$. Define $M_{0}^{\delta}=\Lambda \pi_{i} M$; then $\eta \sigma^{\prime} M_{0}^{\delta}=\Lambda \pi_{i} \sigma M^{\delta}$, and $\eta \sigma^{\prime} M_{0}^{1}, \eta \sigma^{\prime} M_{0}^{2} \Rightarrow 0$.

Let $S_{0}^{\sigma}$ be the sentence obtained from $M_{0}^{\delta}$ by prefixing quantifiers $\forall x_{p q}^{\delta i}$ and $\exists y_{r}^{\delta i}$ in an order such that, if $z$ and $z^{\prime}$ are two of these variables and the term $\eta \sigma^{\prime} z$ is shorter than the term $\eta \sigma^{\prime} z^{\prime}$, then the quantification of $z$ precedes that of $z^{\prime}$. If $p \leqq r$, the term $\eta \sigma^{\prime} x_{p q}^{s i}=\eta x_{p q}^{\delta i}$ is a proper part of the term $\gamma \sigma^{\prime} y_{r}^{\delta i}=s_{r}^{\delta}\left(\eta x_{11}^{\delta i}, \cdots, \gamma_{i} x_{r_{r}}^{\delta i}\right)$, whence $\forall x_{p q}^{\delta i}$ precedes $\exists y_{t}^{\delta i}$ in $S_{0}^{\delta}$. Thus $S_{0}^{\delta}$ arises from $S^{\delta}$ by duplication.

Let $S_{\mathrm{J}}^{\delta}$ have Skolem matrix $\sigma_{0} M_{0}$ where $\sigma_{0} x_{p q}^{\delta i}=x_{p q}^{s i}$ and $\sigma_{0} y_{r}=$ $s_{r}^{\delta i}\left(\cdots, x_{L q}^{\delta j}, \cdots\right)$, the arguments ranging, in order of occurrence in $S_{0}^{\delta}$, over all $x_{p q}^{\delta j}$ that precede $y_{r}$ in $S_{0}^{\delta}$. One has $\eta \sigma^{\prime} x_{p q}^{\delta i}=r_{0} \sigma_{0} x_{p q}^{\delta i}$, but $\eta \sigma^{\prime} y_{r}=s_{r}^{\delta}\left(\gamma_{j} x_{11}^{\delta i}, \cdots, r_{r} x_{r m_{r}}^{\delta i}\right)$ while the term $\eta \sigma_{\nu} y_{r}^{\delta i}=s_{r}^{\delta i}\left(\cdots, \eta x_{p q}^{\delta j}, \cdots\right)$ begins with a different operation symbol and contains additional arguments. To bring these into agreement, define a transformation $\chi$ on terms as follows :

(1) $\chi z=z$ for a variable $z$;

(2) $\chi \eta \sigma^{\prime} y_{r}^{\delta i}=\chi \eta \sigma_{0} y_{r}^{\delta i}$;

(3) for any term $t=w\left(t_{1}, \cdots, t_{\rho(w)}\right)$ not of the form $\eta \sigma^{\prime} y_{r}^{\delta i}$, $\chi t=w\left(\chi t_{1}, \cdots, \chi t_{\rho(w)}\right)$.

The clause (2) if legitimate, by an induction on length of $\eta \sigma^{\prime} y_{r}$. For $\chi \eta \sigma_{0} y_{r}^{\delta i}=s_{r}^{\delta i}\left(\cdots, \chi \eta x_{p q}^{\delta j}, \cdots\right)$ contains $\chi \eta \sigma^{\prime} y_{s}^{\delta k}$ only for those $\chi \eta \sigma^{\prime} y_{r}^{\delta k}$ that occur in some $\chi \eta x_{p q}^{\delta j}$ for $p \leqq r$, and it follows by an induction that for all of these $s<p$. Let $L_{0}$ be the language obtained from $L$ by adjoining the symbols $s_{r}^{\delta i}$. Although neither $\chi$ nor $\chi \eta$ is in general a substitution, when applied to terms of $L_{0}$, which do not contain symbols $s_{r}^{\delta}$, the clause (2) is never invoked; consequently the restriction $\eta_{0}$ of $\chi \eta$ to $L_{0}$ is a substitution.

Since $\eta \sigma^{\prime} M_{0}^{1}, \gamma \sigma^{\prime} M_{0}^{2} \Rightarrow 0$, and $\chi$ induces a transformation on terms, it follows that $\chi \eta \sigma^{\prime} M_{0}^{1}, \chi \eta \sigma^{\prime} M_{0}^{2} \Rightarrow 0$. Now $\chi \eta \sigma^{\prime} y_{r}^{\delta i}=\chi \eta \sigma_{0} y_{r}^{\delta i}$ by definition, while $\sigma^{\prime} x_{p q}^{\delta i}=x_{p q}^{\delta i}=\sigma_{0} x_{p q}^{\delta i}$ implies that $\chi \eta \sigma^{\prime} x_{p q}^{\delta i}=\chi \eta \sigma_{0} x_{p q}^{\delta i}$; it follows that $\chi \eta \sigma^{\prime} M_{0}^{\delta}=\chi \eta \sigma_{0} M_{0}^{\delta}=\eta_{0} \sigma_{0} M_{0}^{\delta}$, the last since $\sigma_{0} M_{0}^{\delta}$ belongs to $L_{0}$. Hence, $\eta_{0} \sigma^{0} M_{0}^{1}, \eta_{0} \sigma_{0} M_{0}^{2} \Rightarrow 0$.

Dropping the subscripts on $S_{0}^{\delta}$, we now have the situation at the beginning of the proof, but with $a=1$, that is with a single substitution $\eta$ such that $\eta \sigma M^{1}, \eta \sigma M^{2} \Rightarrow 0$. From the set of all terms that occur in $\gamma \sigma M^{\delta}$ obtain a set $B^{\delta}$ by deleting successively any term that is expressible, by means of the operation symbols of $L$, in terms of the rest. Since each $\eta \sigma y_{r}^{\delta}=s_{r}^{\delta}\left(\eta x_{11}^{\delta}, \cdots, \eta x_{r m_{r}}^{\delta}\right)$ where $s_{r}^{\delta}$ does not belong to $L$, we 
can suppose that all the $\eta \sigma y_{r}^{\delta}$ belong to $B^{\delta}$. Let $b_{1}^{\delta}, \cdots, b_{a}^{\delta}$ be the remaining elements of $B^{\delta}$. Then for each $x_{p q}^{\delta}$ (that occurs in $M^{\delta}$ ) $r_{p} x_{p q}^{\delta}$ is expressible in terms of the $\eta \sigma y_{r}^{\delta}$ and $b_{h}^{\delta}$. More precisely, if $u_{1}^{\delta}, \cdots, u_{a}^{\delta}$ are new and distinct variables, and $\tau$ a substitution such that $\tau y_{r}^{\delta}=\eta \sigma y_{r}^{\delta}$, $\tau u_{h}^{\delta}=b_{h}^{\delta}$. then there exists in $L$ a term $\theta x_{p q}^{\delta}$ in the variables $y_{r}^{\delta}$ and $b_{h}^{\delta}$ such that $\tau \theta x_{p q}^{\delta}=\eta x_{p q}^{\delta}$. We extend $\theta$ to a substitution by setting $\theta z=z$ for all $z$ other than the $x_{p q}^{1}, x_{p q}^{2}$.

Let $S_{0}^{\delta}$ be the sentence obtained from $\theta M^{\delta}$ by prefixing the quantifiers $\forall u_{h}^{\delta}$ and $\exists y_{r}^{\delta}$ in an order such that if $z$ and $z^{\prime}$ are two of these variables, and $\tau z$ is shorter than $\tau z^{\prime}$, then $z$ precedes $z^{\prime}$ in $S_{0}^{\delta}$. To verify that $S_{0}^{\delta}$ arises from $S^{\delta}$ by specialization, we observe that, for (iii), if $y_{r}^{\delta}$ occurs in $\theta x_{p q}$ then $\tau y_{r}^{\delta}=\eta \sigma y_{r}^{\delta}$ is a proper part of $\tau \theta x_{p q}^{\delta}=r x_{p q}^{\delta}$ whence $r<p$; and, for (iv), if $z$ is any $y_{s}^{\delta}$ or $u_{h}^{\delta}$ and $z$ occurs in $\theta x_{p q}^{\delta}$ for $p \leqq r$, then $\tau z$ is a part of $\eta x_{p q}^{\delta}$ which is in turn a proper part of $\gamma \sigma y_{r}^{\delta}=\tau y_{r}^{\delta}$, whence $z$ precedes $y_{r}^{\delta}$ in $S_{0}^{\delta}$.

Let $S_{0}^{\delta}$ have Skolem matrix $\sigma_{0} \theta M^{\delta}$, where $\sigma_{0} z_{p q}=z$ for all variables $z$ other than the $y_{r}^{\delta}$ and $\sigma_{0} y_{r}^{\delta}=s_{\Delta r}^{\delta}\left(\cdots, u_{h}^{\delta}, \cdots\right)$, the arguments ranging in order over all $u_{h}^{\delta}$ that precede $y_{r}^{\delta}$ in $S_{0}^{\delta}$. From $\eta \sigma M^{1}, \eta \sigma M^{2} \Rightarrow 0$ it remains to construct $\eta_{0}$, one-to-one on the atomic formulas of $\sigma_{0} \theta M^{1}, \sigma_{0} \theta M^{2}$, such that $\eta_{0} \sigma_{0} \theta M^{1}, \eta_{0} \sigma_{0} \theta M^{2} \Rightarrow 0$. For this define a transformation $\chi$ on terms as follows :

(1) $\chi z=z$ for a variable $z$;

(2) $\chi \theta \tau \sigma y_{r}^{\delta}=\chi \tau \sigma_{0} y_{r}^{\delta}$;

(3) for any term $t=w\left(t_{1}, \cdots, t_{\rho(w)}\right.$ not of the form $\tau \theta \sigma y_{r}^{\delta}$,

$$
\chi t=w\left(\chi t_{1}, \cdots, \chi t_{\rho(w)}\right) \text {. }
$$

As in an earlier situation, this definition is legitimate, and the restriction $\eta_{0}$ of $\chi \tau$ to the language $L_{0}$ obtained from $L$ by adjoining the symbols $s_{u r}^{\delta}$ is a substitution. As before we conclude from $\eta \sigma M^{1}, \eta \sigma M^{2} \Rightarrow 0$ that $\chi \tau \theta \sigma M^{1}, \chi \tau \theta \sigma M^{2} \Rightarrow 0$,

Now

$$
\chi \tau \theta \sigma y_{r}^{\varsigma}=\chi \tau \sigma_{0} y_{r}^{\varsigma}=\chi \tau \sigma_{0} \theta y_{r}^{\varsigma}=\eta_{0} \sigma_{0} \theta y_{r}^{\varsigma},
$$

and

$$
\chi \tau \theta \sigma x_{p q}^{\varsigma}=\chi \tau \theta \theta x_{p q}^{\delta}=\chi \tau \theta \sigma_{0} x_{p q}^{\S}=\chi \tau \sigma_{0} \theta x_{p q}^{\delta}=\eta_{0} \sigma_{0} \theta x_{p q}^{\delta} .
$$

It follows that $\chi \tau \theta \sigma M^{\delta}=\eta_{0} \sigma_{0} \theta M^{\delta}$, whence

$$
\eta_{0} \sigma_{0} \theta M^{1}, \eta_{0} \sigma_{0} \theta M^{2} \Rightarrow 0
$$

It remains to show that $\eta_{0} \sigma_{0}=\chi \tau \sigma_{0}$ is one-to-one on the terms of each $\theta M^{\delta}$. We show first that $\tau \theta \sigma$ is one-to-one on such terms. These terms are terms in the variables $u_{h}^{\delta}$ and $y_{r}^{\zeta}$, containing only the operation symbols of $L$. Note that $\tau \theta \sigma u_{h}^{\delta}=\tau \theta u_{h}^{\delta}=\tau u_{h}^{\delta}=b_{h}^{\delta}$ and $\tau \theta \sigma y_{r}^{\delta}=\eta \sigma y_{r}^{\delta}$. 
From the construction of $B$, it follows that, for two such terms $t$ and $t^{\prime}, \tau \theta \sigma t=\tau \theta \sigma t^{\prime}$ cannot hold for one of $t, t^{\prime}$ a variable unless $t=t^{\prime}$. Suppose now that $t=w\left(t_{1}, \cdots, t_{\rho(w)}\right)$ and $t^{\prime}=w^{\prime}\left(t_{1}^{\prime}, \cdots, t_{\rho\left(w^{\prime}\right)}^{\prime}\right)$. Comparing the first symbols we conclude from $\tau \theta \sigma t=\tau \theta \sigma t^{\prime}$ that $w=w^{\prime}$, and the arguments agree :

$$
\tau \theta \sigma t_{1}=\tau \theta \sigma t_{i}^{\prime} \quad i=1, \cdots, \rho(w)=\rho\left(w^{\prime}\right) .
$$

By induction on the length of the shorter of $t, t^{\prime}$ we conclude that each $t_{i}=t_{i}^{\prime}$, whence $t=t^{\prime}$.

Finally, $\chi \tau \sigma_{0} y_{r}^{\delta}=\chi \tau \theta \sigma y_{r}^{\delta}$ by definition, and $\chi \tau \sigma_{0} u_{h}^{\delta}=\chi \tau u_{h}^{\delta}=\gamma \tau \theta \sigma u_{h}^{\delta}$. Hence $\chi \tau \sigma_{0}=\chi \tau \theta \sigma$ on terms of $\theta M^{\delta}$. But $\chi$ is evidently one-to-one on terms that do not contain the symbols $s_{\mathrm{v} r}^{\delta}$. Hence, for terms $t$ and $t^{\prime}$ of $\theta M^{\delta}, \sigma \tau \sigma_{0} t=\chi \tau \sigma_{0} t^{1}$ implies $\chi \tau \theta \sigma t=\chi \tau \theta \sigma t^{\prime}$, hence $\tau \theta \sigma t=\tau \theta \sigma t^{\prime}$, and, by the property of $\tau \theta \sigma$ established above, $t=t^{\prime}$. This completes the proof of the Main Theorem.

5. The Interpolation theorem. Let $S$ and $T$ be sentences of a language $L$ such that $S \Rightarrow T$. Then there exists a sentence $S^{0}$ of the language $L$ such that $S \Rightarrow S^{0}, S^{0} \Rightarrow T$, and that a relation symbol occurs positively in $S^{0}$ only if it occurs positively in both $S$ and $T$, and occurs negatively in $S^{0}$ only if it occurs negatively in both $S$ and $T$.

Proof. $S$ is equivalent to a prenex sentence $S^{1}$ such that a relation symbol occurs positively (negatively) in $S^{1}$ only if it occurs positively (negatively) in $S$. And $\sim T$ is equivalent to a prenex sentence $S^{2}$ such that a relation symbol occurs positively (negatively) in $S^{2}$ only if it occurs negatively (positively) in $T$. Since $S^{1}, S^{2} \Rightarrow 0$, by the Main Theorem there exist prenex sentencs $U^{1}$ and $U^{2}$ such that $S^{1} \Rightarrow U^{1}, S^{2} \Rightarrow U^{2}$, that $U^{1}$ contains the same kinds of occurrences of relation symbols as $S^{1}$ and $U^{2}$ as $S^{2}$, and that $\eta \sigma M^{1}, \eta \sigma M^{2} \Rightarrow 0$ where $\sigma M^{1}, \sigma M^{2}$ are the Skolem matrices of $U^{1}, U^{2}$, and $\eta$ is a substitution that is one-to-one on the atomic formulas of each of $\sigma M^{1}, \sigma M^{2}$. All this is not altered if we modify $U^{1}$, $U^{2}$ by reducing $M^{1}, M^{2}$ to normal form.

It will suffice to find $S^{0}$ such that $U^{1} \Rightarrow S^{0}$, and $S^{0}, U^{2} \Rightarrow 0$, and a relation symbol occurs positively (negatively) in $S^{0}$ only if it occurs positively (negatively) in $U^{1}$ and negatively (positively) in $U^{2}$. Write $M^{\delta}=$ $\vee M_{i}^{\delta}$, each $M_{i}^{\delta}=\wedge M_{i j}^{\delta}$, and each $M_{i j}^{\delta}$ either $A_{i j}^{\delta}$ or $\sim A_{i j}^{\delta}$ where $A_{i j}^{\delta}$ is an atomic formula. Define $M^{0}=\mathrm{V} M_{i}^{0}$ where $M_{i}^{0}=0$ if $M_{i}^{1} \Rightarrow 0$, and otherwise $M_{i}^{0}$ results from $M_{i}^{1}$ by deleting all $M_{i j}^{1}$ such that $\sim \eta \sigma M_{i j}^{1}$ is not equivalent to some $\eta_{\sigma} M_{h k}^{2}$. Let $S^{0}$ be the sentence obtained from $U^{1}$ by replacing its matrix $M^{1}$ by the matrix $M^{0}$. It is immediate that the occurences of relation symbols in $S^{0}$ are related to those in $U^{1}$ and $U^{2}$ in the required manner. Moreover. since $M^{1} \Rightarrow M^{0}$ is immediate, it follows easily that $U^{1} \Rightarrow S^{0}$. 
It remains to show that $S^{0}, U^{2} \Rightarrow 0$, and for this it will suffice to to show that $\eta \sigma M^{0}, \eta \sigma M^{2} \Rightarrow 0$. Since $\eta \sigma M^{1} \wedge \eta \sigma M^{2} \Rightarrow 0$, then for all $i, h$, $\eta \sigma M_{i}^{1} \wedge \eta \sigma M_{h}^{2} \Rightarrow 0$. We want to conclude that for all $i, h, \eta \sigma M_{i}^{0} \wedge \eta \sigma M_{h}^{2} \Rightarrow 0$. Since $\sigma$ is clearly one-to-one on the terms of $M^{1}$, so is $\eta \sigma$, and $\eta \sigma M_{i}^{1} \Rightarrow 0$ implies $M_{i}^{1} \Rightarrow 0$, whence by definition $M_{i}^{0}=0$, hence $\eta \sigma M_{i}^{0}=0$ and the conclusion follows. If $\eta \sigma M_{h}^{2} \Rightarrow 0$ the conclusion is immediate. In the remaining case there exist $j$ and $k$ such that $\sim \eta \sigma M_{i j}^{1} \Leftrightarrow \eta \sigma M_{h k}^{2}$. But then, by definition, $M_{i}^{0}$ still contains the conjunct $M_{i j}^{1}$, and again $\eta \sigma M_{i}^{0} \wedge \eta \sigma M_{h}^{2} \Rightarrow 0$. Since $\eta \sigma M_{i}^{0} \wedge \eta \sigma M_{h}^{2} \Rightarrow 0$ for all $i, h$, it follows that $\eta \sigma M^{0} \wedge \eta \sigma M^{2} \Rightarrow 0$, completing the proof.

It was stated in the introduction that the Interpolation Theorem remains true for the predicate calculus with identity. Precisely, we restrict the definition of a language to apply only to those that contain a fixed relation symbol $e$ of rank two, and the definition of interpretation to admit only those $\mu$ for which $\mu e$ is the identity relation on the domain of $\mu$. The relation $S \Rightarrow T$ then acquires a stronger meaning. Nonetheless, the Interpolation Theorem as stated remains true in this new sense. (It may be well to note that $e$ is included among the relation symbols mentioned in the conclusion of the theorem.) In fact, all statements in this paper remain true in the new sense, apart from two modifications. First, Proposition 1 must be modified by enlarging $J$ to contain (the coset of) each formula $e(t, t), t$ a term, and to contain any formula $F^{\prime}$ obtainable from a formula $F$ in $J$ by replacing an occurrence of a term $t$ by a new term $t^{\prime}$, provided that $e\left(t, t^{\prime}\right)$ is in $J$. Second, in the proof of the Interpolation Theorem, the $M_{i}^{\delta}$ as described above must be similarly enlarged by adjoining to each the finite set of all $M_{i j}^{\delta}$ of the form $A$ or $\sim A, A$ atomic, such that $M_{i}^{\delta} \Rightarrow M_{i j}^{\delta}$ in the present sense.

The Interpolation Theorem can be refined in other ways. Conditions can be imposed on the internal structure of the atomic formulas $r\left(t_{1}, \cdots, t_{\rho(r)}\right)$ containing the relation symbol $r$. For example, define an $I$-occurrence of $r$ in $S$ to be one in which each $t_{i}$, for $i \in I \subseteq\{1, \cdots \rho(r)\}$, is a variable universally quantified in $S$. Then it can be required that $r$ have $I$-occurrences is $S^{0}$ only if it has $I^{\prime}$-occurrences in $S$ and $I^{\prime \prime}$-occurrences in $T$, where $I^{\prime \prime} \subseteq I \subseteq I^{\prime}$. Alternatively, stronger conditions can be imposed on the external context in which a relation symbol occurs. For example, suppose all positive occurrences in $S$ of a relation symbol $r$ are in formulas $A^{\prime} \supset A$ where $A$ and $A^{\prime}$ are atomic formulas, and that none of the relation symbols appearing in the parts $A^{\prime}$ of these formulas have positive occurrences in $S$, except possibly in parts $A$; then $S^{0}$ can be required to contain no positive occurrences of $r$. Such refinements of the Interpolation Theorem have proved useful in the study of homomorphisms and subdirect products of models, but because of their special nature it does not seem worthwhile to give separately formal statements and proofs of these results. 


\section{REFERENCES}

1. E. W. Beth, On Padoa's method in the theory of definition, Indag, Math. 15 (1953), 66. 2. - . The Foundations of Mathematics, North Holland, Amsterdam, 1958.

3. W. Craig, Linear reasoning. A new form of the Herbrand-Gentzen theorem, J. Symbolic Logic 22 (1957), 250.

4. , Three uses of the Herlrand-Gentzen theorem in relating model theory and proof theory, J. Symbolic Logic 22 (1957), 269.

5. G. Gentzen, Untersuchungen über das logische Schliese€n, Math. Z. 39 (1934-35), 176, 405.

6. K. Gödel, Die Vollständigkeit der Axicme des logischen Funktionenkalküls, Monatsh. Math. Phys. 37 (1930), 349.

7. L. Henkin, The completeness of the first order functional calculus, J. Symbolic Logic 14 (1949), 159.

8. J. Herbrand, Recherches sur la théorie de la démonstration, Travaux Soc. Sci. et Let. Varsovie, Cl. III, No. 33, 1930.

9. S. C. Kleene, Introduction to Metamathematics, Van Nostrand, New York, 1952.

10. A. Robinson, A result on consistency and its application to the theory of definition, Indag. Math. 18 (1956), 47.

11. A Tarski, Some notions and mcthods on the borderline of algebra and metamathe. matics, Proc. International Congress of Math. 1, Cambridge, 1950, 705.

12. A. Tarski, Contributions to the theory of models, I, II, III, Indag. Math. 16 (1954) 572,$582 ; 17$ (1955), 55.

\section{UNIVERSITY OF MICHIGAN}




\section{PACIFIC JOURNAL OF MATHEMATICS}

\section{EDITORS}

\section{David Gilbarg}

Stanford University

Stanford, California

R. A. Beaumont

University of Washington

Seattle 5 , Washington
A. L. Whiteman

University of Southern California

Los Angeles 7, California

L. J. PAIGE

University of California

Los Angeles 24, California

\section{ASSOCIATE EDITORS}
E. F. BECKENBACH
C. E. BURGESS
E. HEWITT
A. HORN

\author{
V. GANAPATHY IYER \\ R. D. JAMES \\ M. S. KNEBELMAN \\ L. NACHBIN
}
I. NIVEN
E. G. STRAUS
T. G. OSTROM
H. L. ROYDEN
G. SZEKERES
M. M. SCHIFFER
F. WOLF
K. YOSIDA

\section{SUPPORTING INSTITUTIONS}

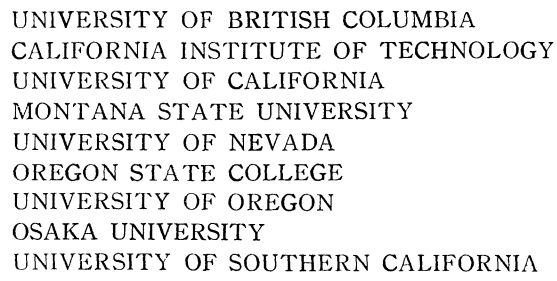

UNIVERSITY OF BRITISH COLUMBIA CALIFORNIA INSTITUTE OF TECHNOLOGY UNIVERSITY OF CALIFORNIA MONTANA STATE UNIVERSITY UNIVERSITY OF NEVADA OREGON STATE COLLEGE UNIVERSITY OF OREGON OSAKA UNIVERSITY UNIVERSITY OF SOUTHERN CALIFORNIA

STANFORD UNIVERSITY

UNIVERSITY OF TOKYO

UNIVERSITY OF UTAH

WASHINGTON STATE COLLEGE

UNIVERSITY OF WASHINGTON

AMERICAN MATHEMATICAL SOCIETY CALIFORNIA RESEARCH CORPORATION HUGHES AIRCRAFT COMPANY SPACE TECHNOLOGY LABORATORIES

Printed in Japan by Kokusai Bunken Insatsusha (International Academic Printing Co., Ltd.), Tokyo, Japan 


\section{Pacific Journal of Mathematics}

\section{Vol. 9, No. 1 \\ May, 1959}

Julius Rubin Blum and Murray Rosenblatt, On the structure of infinitely

divisible distributions . ............................. 1

Robert Geroge Buschman, Asymptotic expressions for

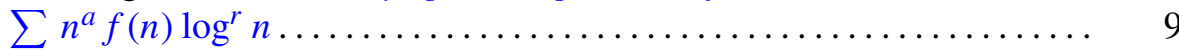

Eckford Cohen, A class of residue systems $(\bmod r)$ and related arithmetical

functions. I. A generalization of Möbius inversion .............. 13

Paul F. Conrad, Non-abelian ordered groups ................... 25

Richard Henry Crowell, On the van Kampen theorem............... 43

Irving Leonard Glicksberg, Convolution semigroups of measures ........ 51

Seymour Goldberg, Linear operators and their conjugates ............ 69

Olof Hanner, Mean play of sums of positional games .............. 81

Erhard Heinz, On one-to-one harmonic mappings ................ 101

John Rolfe Isbell, On finite-dimensional uniform spaces . . ........... 107

Erwin Kreyszig and John Todd, On the radius of univalence of the function

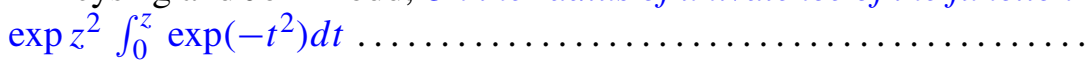

Roger Conant Lyndon, An interpolation theorem in the predicate

calculus......................................... 129

Roger Conant Lyndon, Properties preserved under homomorphism ........ 143

Roger Conant Lyndon, Properties preserved in subdirect products ....... 155

Robert Osserman, A lemma on analytic curves ................ 165

R. S. Phillips, On a theorem due to Sz.-Nagy..................... 169

Richard Scott Pierce, A generalization of atomic Boolean algebras ....... 175

J. B. Roberts, Analytic continuation of meromorphic functions in valued fields................................. 183

Walter Rudin, Idempotent measures on Abelian groups ................ 195

M. Schiffer, Fredholm eigen values of multiply-connected domains ........ 211

V. N. Singh, A note on the computation of Alder's polynomials ......... 271

Maurice Sion, On integration of 1-forms ...................... 277

Elbert A. Walker, Subdirect sums and infinite Abelian groups........... 287

John W. Woll, Homogeneous stochastic processes . .................. 293 